\title{
Skills Assessment Module
}

The Skills Assessment Module (SAM) is a battery of tasks designed to assess the affective, cognitive, and psychomotor abilities of special needs vocational students. SAM is designed to be used with mildly handicapped and disadvantaged youths, 14-18 years of age. Data generated by SAM is used to identify vocational educational needs of the student, thus facilitating design of a vocational program to meet that student's individual needs. Test developers emphasize that SAM should not be used to predict job or training program performance but rather to help design the student's vocational program.

SAM requires one to three hours to administer and consists of three pencil-and-paper tests and twelve hands-on tasks. In addition, the test administrator must rate the student on eight work behaviors. The pencil-and-paper tests include a learning styles inventory, a revised Beta Exam (a nonverbal measure of intellectual ability), and an auditory directions screening test. The twelve hands-on tasks include testing of the following.

1. Digital discrimination

2. Clerical, measurement, and numerical skills

3. Motor coordination

4. Aiming

5. Manual and finger dexterity

6. Form, spatial, and color perception

7. Ability to follow written and diagrammatic instructions.

Behavior observations are made throughout the testing procedure. The student is rated on a scale of 1 to 5 ( $1=$ superior, $5=$ needs improvement) as to appearance, communication skills, conformity to rules, endurance, initiative, interpersonal traits, reactions to assigned tasks, and safety consciousness.

All equipment needed for the testing is contained in a wooden custom-made case ( 1.5 feet $\times 1.5$ feet $\times 1$ foot) that is portable but, according to the vocational counselors to whom I spoke, considered somewhat heavy (44 pounds). The cost for the test is $\$ 1695$.

SAM can be scored by hand or computer; the scoring software is compatible with either Apple or IBM computers. The software also includes a Vocational Perfomance Matrix and a Local Norm Development System. The Vocational Performance Matrix correlates the student's performance with the skills required for 15 different vocational categories. This correlational information may be useful in providing career counseling to special needs vocational students who request direction. The Local Norm Development System assists the vocational program in collecting normative data on their students.

A procedure manual accompanies the test. The manual is clearly written and contains information about the purpose of each task, a description of the materials used and the activity to be performed, verbal instructions to be read to the student, and instructions for the scoring procedure and interpretation of the data. The manual was well organized with good illustrations.

Normative data was collected for SAM from six vocational high schools in urban, suburban, and rural sites from three groups of students: average vocational high school students, mildly handicapped students, and disadvantaged students. The mildly handicapped group included students who were mildly mentally retarded and had behavior disorders, learning disabilities, or were mildly physically handicapped. The disadvantaged group included students who were economically or academically disadvantaged. A total of 360 students were included in the norming study with an equal representation from each of the three groups.

A study of test-retest or intra-rater reliability

WORK 1992; 2(2):85-86

Copyright (C) 1992 by Andover Medical Publishers, Inc. 
is reported in the procedure manual but has not been published in a refereed journal. This reliability study involved 50 students ( 25 male, 25 female), ages 14-18, who took the test twice over a three-to-five-day period. Each of the hands-on tasks were evaluated separately for reliability using Pearson's $r$ statistic. Pearson's $r$ correlation coefficients ranged from $0.80-0.95$, indicating high to very high correlation between scores from the first test to the second. Inter-rater reliability has not been addressed.

The tasks included in SAM were rated according to importance in a vocational program by vocational counselors, and in this way was shown to have a degree of content validity. Other more rigorous testing of validity has not been carried out. The test publisher stated that predictive validity had not been studied because of a desire to avoid using the test to predict student performance or employability. However, discriminant validity may be a more important type of validity to be studied in this instance since the purpose of SAM is to identify areas of weakness for intervention. Discriminant validity could be easily shown by comparing the three groups used in the norming process. If SAM has discriminant validity, a significant difference should be present between the average high school student and the mildly handicapped students. Test developers have made a good initial effort at establishing reliability and validity of SAM but must carry such testing further in order to adequately document reliability and validity.

In summary, SAM appears to be a carefully and thoughtfully developed test of the affective, cognitive, and psychomotor abilities of vocational students with special needs. The test is designed to identify needs and not to predict performance. SAM has been normed on 360 students and some limited reliability and validity studies have been carried out.

Deborah Lechner, MS, PT 